\title{
In vitro Bio-Efficacy of Botanicals and Organic Amendments against Dry Root Rot of Safflower
}

\author{
P.N. Gawande ${ }^{1}$, V.M. Gholve ${ }^{1 *}$ and S.B. Ghuge ${ }^{3}$ \\ ${ }^{1}$ Department of Plant Pathology, Sorghum Research Station, ${ }^{2}$ Incharge Safflower Research \\ Station, V.N. Marathwada Krishi Vidyapeeth, Parbhani - 431 402, Maharashtra, India \\ *Corresponding author
}

\section{A B S T R A C T}

\section{Keywords \\ Dry root rot, Safflower, Botanicals, Organic amendments, Management, Macrophomina phaseolina \\ Article Info \\ Accepted: \\ 15 October 2018 \\ Available Online: \\ 10 November 2018}

\begin{abstract}
Among the diseases of safflower; dry root rot disease caused by Macrophomina phaseolina (Tassi.) Goid is one of the most destructive and wide spread disease of Safflower (Carthamous tinctorius L.) causing accountable losses of about 25-60 per cent. Eleven Botanicals evaluated in vitro were found fungistatic/antifungal against $M$. phaseolina. Amongs these A. sativum followed by $A$. cepa and V. negundo caused significantly highest mean mycelial inhibition 83.57, 72.09 and 65.85 per cent respectively. Total eight organic amendments tested in pot culture, out of which Neem seed cake, cotton seed cake and Groundnut cake (each @ $50 \mathrm{gm} / \mathrm{kg}$ soil) recorded average mortality reduction of 74.30, 67.22 and 57.57 per cent respectively in safflower cv. Bhima.
\end{abstract}

\section{Introduction}

Safflower (Carthamous tinctorius L.) commonly known as Kardi (Marathi), Kusube (Kannada), Kusum (Hindi) and Kusumba (Telugu) is one of the important Rabi oilseed crop of the country originated from Abyssinia and Afghanistan. It is drought tolerant, selfpollinated crop belonging to the family compositae or asteraceae. The world area under safflower is 8.22 lakh ha with 5.83 lakh tones production and productivity of 709 $\mathrm{kg} / \mathrm{ha}$. India is in first place in terms of area and production of safflower in the world. In India safflower is raised over an area of 296.0lakh ha with a production of 180.0 lakh tones. The important states growing safflower are Maharashtra, Karnataka, Andhra Pradesh, Madhya Pradesh, Gujarat, Orissa and Bihar. In Maharashtra it has occupied an area of 193 lakh ha with a production of 114 lakh tones and productivity of $510 \mathrm{~kg} / \mathrm{ha}$ (Anonymous 2013-2014).

Among the diseases of safflower; dry root rot disease caused by Macrophomina phaseolina (Tassi.) Goid is one of the most destructive and wide spread diseases of Safflower (Carthamous tinctorius L.) causing accountable losses of about 25-60 per cent. It is the major soil borne disease and appears sporadically all over the country 
(Shambharkar and Indi, 1987). Occurrence of this disease on safflower in India was first reported by Amarsingh and Bhowmik (1979) from IARI, New Delhi and later on by others from different parts of the country (Kore and Deshmukh, (1982) and Lukade, (1992) from Maharashtra; Singh et al., (1987) from M.P). The pathogen Macrophomina phaseolina has been reported to infect safflower at various growth stages of the plant. Affected plants showed grayish black discoloration at collar region from where the plant later broke. The lateral roots were turned black and their skin was easily sloughed off exposing the inner cortical tissues. Affected portion was covered with small, numerous, black sclerotia. These symptoms were more prominent after flowering and their severity increased at maturity. Above ground appearance of disease plant was expressed with yellow, dried leaves, finally wilting the plant completely which could easily be pulled out from soil (Amarsingh and Bhowmik, 1979). It has therefore become highly essential to have a good knowledge of such association, method of detection as well as way to control them. Considering economic importance of the disease, the present investigation was undertaken.

\section{Materials and Methods}

\section{In vitro evaluation of botanicals / plant extracts}

Aqueous extracts of 11 botanicals viz., Mehandi, Ginger, Onion, Tulsi, Nirgudi, Neem, Garlic, Turmeric, Adulsa, Shatawari and Noni extract were evaluated in vitro against $M$. phaseolina, applying poisoned food technique. Aqueous extracts of the test botanicals were prepared by grinding with mixture-cum grinder. The $100 \mathrm{gm}$ washed leaves/ bulbs/rhizomes of each of the test botanicals were macerated in $100 \mathrm{ml}$ distilled water (w/v) separately and the macerates obtain were filtered through double layered muslin cloth. Each of the filtrate obtained was further filtered through Whatman No. 1 filter paper using funnel and volumetric flasks (100 $\mathrm{ml}$ caps.). The final clear extracts/filtrates obtained formed the standard aqueous extract of 100 per cent concentration. These were evaluated (@10,15 and 20\% each) in vitro against $M$. phaseolina, applying Poisoned food technique (Nene and Thapliyal, 1993) and using Potato dextrose agar (PDA) as basal culture medium. An appropriate quantity of each test aqueous extract (100\%) was separately mixed thoroughly with autoclaved and cooled $\left(40^{\circ} \mathrm{C}\right)$ PDA medium in conical flasks (250 $\mathrm{ml}$ cap.) to obtain desired concentrations (@10,15 and 20\%). The PDA medium amended separately with the test aqueous extract was then poured $(20 \mathrm{ml} /$ plate $)$ into sterile glass Petri plates (90 $\mathrm{mm}$ dia.) and allowed to solidify at room temperature. For each test botanical extract and their respective concentrations, three replications were maintained. Upon solidification of the PDA (amended), all the treatment plates were aseptically inoculated by placing in the centre a $5 \mathrm{~mm}$ mycelial disc obtained from a week old actively growing pure culture of $M$. phaseolina. Plates containing plain PDA without any botanical extract and inoculated with mycelial disc of the test pathogen served as untreated control. All these plates were then incubated at $28+2^{0} \mathrm{C}$ temperature for a week or till the untreated control plates were fully covered with mycelial growth of the test pathogen.

Observations on radial mycelial growth/colony diameter of the test pathogen were recorded treatment wise at 24 hours interval and continued till mycelial growth of the test pathogen was fully covered in the untreated control plates. Per cent inhibition of mycelial growth of the test pathogen over untreated control was calculated (Vincent, 1927). 
In vitro evaluation of organic amendments (pot culture)

A total of 08 amendments viz., Neem cake, Caster cake, Groundnut cake, Cotton seed cake, Sunflower cake, FYM/Compost, Poultry manure and Vermicompost were evaluated against $M$. phaseolina by soil application and sick soil method in pot culture under screen house conditions. Except Vermicompost all of the tests amendments were grind physically to rough powder and used for soil application. Earthen pots $(30 \mathrm{~cm}$ dia.) filled with potting mixture (soil + sand + FYM) were inoculated (@ $50 \mathrm{~g} / \mathrm{kg}$ mixture) with the test pathogen culture multiplied on sand: maize medium, watered adequately and incubated in screen house for 10 days to proliferate the pathogen in pots (sick soil).

Then all the test amendments were applied (@ $50 \mathrm{~g} / \mathrm{kg}$ mixture) to the earthen pots containing sick soil potting mixture, mixed thoroughly, watered regularly and maintained in screen house. After $72 \mathrm{hrs}$ of amendments application, surface sterilized $\left(0.1 \% \mathrm{HgCl}_{2}\right)$ healthy seeds of safflower cv. Bhima were sown $(10 \mathrm{seed} /$ pot), watered adequately and maintained in the screen house. Three pot /treatment /replication were maintained. In this method of soil application of the amendments, the earthen pots containing M. phaseolina sick soil and sown with surface sterilized healthy seed of susceptible Safflower cv. Bhima was maintained as untreated (without any amendment) control.

Observations in experiment were recorded on seed germination and pre-emergence seed rot (PESR) were recorded at 7 days after sowing and that of post-emergence seedling mortality (PESM) were recorded at 30 days after sowing. The percentage seed germination, preemergence seed rot (PESR) and postemergence seedling mortality (PESM) were calculated by following formulae.
No. of seeds germinated

Germination $(\%)$ = --------------------- x 100

Total no. of seeds sown

No. of seeds ungerminated

$\begin{aligned} \operatorname{PESR}(\%)= & \text {----------------------- x } 100 \\ & \text { Total no. of seeds sown }\end{aligned}$

No. of seedlings died

$\operatorname{PESM}(\%)=-\frac{\text {--------------------- x } 100}{\text { Total no. of seedlings }}$

C-T

Reduction (\%) in PESR \& PESM = ----- x 100

$\mathrm{C}$

Where,

$\mathrm{C}=$ Per cent rot $/$ mortality in treatment pots

$\mathrm{T}=$ Per cent rot $/$ mortality in untreated control pots

\section{Results and Discussion}

\section{In vitro evaluation of plant extracts / botanicals}

Aqueous extracts of 11 botanicals were evaluated in vitro (each@10,15 and 20\%) against $M$. phaseolina and the results obtained on its mycelial growth and inhibition are presented in the Table 3 and depicted in Figure 1, 2, 3 and PLATE III. Results (Table 3) revealed that all the 11 botanicals extracts tested were fungistatic / antifungal to $M$. phaseolina, which significantly reduced mycelial growth and increased its inhibition over untreated control. The mycelial growth was found to be decreased and its inhibition was increased with increase in concentrations of the botanicals tested.

\section{Mycelial growth}

At 10 per cent, (Table 1, Fig. 1, PLATE -I), radial mycelial growth of the test pathogen was ranged from $19.66 \mathrm{~mm}$ (A. sativam) to 
$82.00 \mathrm{~mm}$ (A. vasica), $82.00 \mathrm{~mm}$ (M. citrofolia), both were at par, as against 90.00 $\mathrm{mm}$ in untreated control. However, significantly least mycelial growth was recorded with $A$. sativam $(19.66 \mathrm{~mm})$. This was followed by the botanicals viz., A. cepa (30.66mm), $V$. negundo $(34.00 \mathrm{~mm}), Z$. officinale $(36.00 \mathrm{~mm})$, A. indica $(43.00 \mathrm{~mm})$, and $O$. sanctum $(54.66 \mathrm{~mm}), L$. innermis $(55.33 \mathrm{~mm})$, both were at par. These were followed by the botanicals viz., C. longa (61.33mm), A. racemosa (79.66mm). Botanicals $M$. citrofolia $(82.00 \mathrm{~mm})$ and $A$. vasica $(82.00 \mathrm{~mm})$ showed highest radial mycelial growth and least effective against $M$. phaseonlina.

At 15 per cent (Table 1, Fig. 2, PLATE -I), radial mycelial growth of the test pathogen was ranged from $14.33 \mathrm{~mm}$ (A. sativam) to $78.66 \mathrm{~mm}$ (A. vasica), as against $90.00 \mathrm{~mm}$ in untreated control. However, significantly least mycelial growth was recorded with $A$. sativam (14.33mm).

This was followed by the botanicals viz., A. cepa $(26.33 \mathrm{~mm}), V$. negundo $(31.00 \mathrm{~mm}), Z$. officinale $(32.66 \mathrm{~mm}), A$. indica $(35.66 \mathrm{~mm})$, and $O$. sanctum $(43.33 \mathrm{~mm})$ and $L$. innermis $(51.00 \mathrm{~mm})$.This was followed by the botanicals viz., $C$. longa $(58.33 \mathrm{~mm}), A$. racemosa $(70.66 \mathrm{~mm})$, and $M$. citrofolia (71.33mm). Botanical A. vasica $(75.33 \mathrm{~mm})$ showed highest radial mycelial growth.

At 20 per cent, (Table 1, Fig. 3, PLATE -I) radial mycelial growth of the test pathogen was ranged from $10.33 \mathrm{~mm}$ (A. sativam) to $75.33 \mathrm{~mm}$ (A. vasica), as against $90.00 \mathrm{~mm}$ in untreated control. However, significantly least mycelial growth was recorded with $A$. sativam $(10.33 \mathrm{~mm})$. This was followed by the botanicals viz., A. cepa $(18.33 \mathrm{~mm}), \quad V$. negundo $(27.33 \mathrm{~mm})$, Z. officinale $(29.66 \mathrm{~mm})$, A. indica $(30.66 \mathrm{~mm})$, both were at par. This was followed by $O$. sanctum $(41.33 \mathrm{~mm})$, and
L. innermis $(47.00 \mathrm{~mm})$. This was followed by the botanicals viz., C. longa $(56.00 \mathrm{~mm}), A$. racemosa $(70.66 \mathrm{~mm})$ and $M$. citrofolia $(71.00 \mathrm{~mm})$ and $A$. vasica $(75.33 \mathrm{~mm})$ showed highest radial mycelial growth.

\section{Mycelial growth inhibition}

Results obtained on mycelial growth inhibition of the test pathogen with the botanicals tested at various concentrations are presented in the Table 1 and depicted in the PLATE I. Results (Table 1) revealed that all the botanicals tested (@each 10, 15 and 20\%), significantly inhibited mycelial growth of the test pathogen over untreated control. Further, it was found that percentage mycelial growth inhibition of the test pathogen was increased with increase in concentrations of the botanicals tested (PLATE I).

At 10 per cent (Table 1, Fig. 1 and PLATE -I) mycelial growth inhibition was ranged from 8.88 (A. vasica) to 78.14 (A. sativam) per cent. However, significantly highest mycelial growth inhibition was recorded with $A$. sativam $(78.14 \%)$ and A. cepa $(65.92 \%)$. This was followed by the botanicals viz., $V$. negundo (62.39\%), Z. officinale (59.99\%), A. indica (52.22\%). Botanical $O$. sanctum (39.25\%), and L. innermis $(38.51 \%)$ were at par. This was followed by $C$. longa $(31.85 \%)$ and A. racemosa (11.48\%). Botanicals viz., $M$. citrifolia $(8.88 \%)$, and A. vasica $(8.88 \%)$ both were found comparatively less effective with less than 20 per cent growth inhibition.

At 15 per cent (Table 1, Fig. 2 and PLATE-I), mycelial growth inhibition was ranged from 12.59 (A. vasica) to 84.07 (A. sativam) per cent. However, significantly highest mycelial growth inhibition was recorded with $A$. sativam (84.07\%) and A. cepa (70.73\%). These were followed by the botanicals viz., $V$. negundo (65.55\%), Z. officinale (63.70\%), A. indica $(60.37 \%)$. The botanical $O$. sanctum 
(48.51\%), L. innermis (43.33\%), C. longa (35.14\%) were at par. Botanical A. racemosa (18.14\%). M. citrifolia (15.18\%), and $A$. vasica (12.59\%) were found comparatively less effective with less than 20 per cent growth inhibition.

At 20 per cent (Table 1, Fig. 3 and PLATE-I) mycelial growth inhibition was ranged from 16.29 (A. vasica) to 88.51 (A. sativam) per cent. However, significantly highest mycelial growth inhibition was recorded with $A$. sativam $(88.51 \%)$ and $A$. cepa $(79.62 \%)$. This was followed by the botanicals viz., $V$. negundo (69.62\%), Z. officinale (67.03\%), A. indica (65.92\%). botanicals $O$. sanctum $(54.7 \%)$ and L. innermis $(47.77 \%)$, were at par. This was followed by C. longa (37.77\%), A. racemosa $(21.48 \%)$ and $M$. citrifolia $(21.11 \%)$, both were at par and A. vasica (16.29\%) were found comparatively less effective with less than 20 per cent growth inhibition.

Thus, on the basis of mycelial growth inhibition caused by the test botanicals and those found most antifungal against $M$. phaseolina were A. sativum, A. cepa, $V$. negundo, Z. officinale, A. indica, $O$. sanctum, L. innermis, $C$. longa, and $A$. racemosa.

Table.1 In vitro efficacy of botanicals against mycelial growth and inhibition of $M$. phaseolina

\begin{tabular}{|c|c|c|c|c|c|c|c|}
\hline \multirow{2}{*}{$\begin{array}{l}\text { Tr. } \\
\text { No. }\end{array}$} & \multirow[t]{2}{*}{ Treatments } & \multicolumn{3}{|c|}{ Mean colony Dia.(mm)* at Conc. } & \multicolumn{3}{|c|}{ \% Inhibition at Conc. } \\
\hline & & $10 \%$ & $15 \%$ & $20 \%$ & $10 \%$ & $15 \%$ & $20 \%$ \\
\hline$\overline{T_{1}}$ & $\begin{array}{l}\text { Mehandi } \\
\text { (L. innermis) }\end{array}$ & 55.33 & 51.00 & 47.00 & $\begin{array}{c}38.51 \\
(22.65)\end{array}$ & $\begin{array}{l}43.33 \\
(25.67)\end{array}$ & $\begin{array}{l}47.77 \\
(28.53)\end{array}$ \\
\hline $\mathbf{T}_{2}$ & $\begin{array}{l}\text { Ginger } \\
\text { (Z.officinale) }\end{array}$ & 36.00 & 32.66 & 29.66 & $\begin{array}{c}59.99 \\
(36.86)\end{array}$ & $\begin{array}{l}63.70 \\
(39.56)\end{array}$ & $\begin{array}{c}67.03 \\
(42.08)\end{array}$ \\
\hline $\mathbf{T}_{\mathbf{3}}$ & $\begin{array}{l}\text { Onion } \\
(A . \text { cepa })\end{array}$ & 30.66 & 26.33 & 18.33 & $\begin{array}{c}65.92 \\
(41.23)\end{array}$ & $\begin{array}{c}70.73 \\
(45.02)\end{array}$ & $\begin{array}{c}79.62 \\
(52.77)\end{array}$ \\
\hline $\mathbf{T}_{4}$ & $\begin{array}{l}\text { Tulsi } \\
\text { (O. sanctum) }\end{array}$ & 54.66 & 46.33 & 41.33 & $\begin{array}{l}39.25 \\
(23.11)\end{array}$ & $\begin{array}{l}48.51 \\
(29.02)\end{array}$ & $\begin{array}{l}54.07 \\
(32.73)\end{array}$ \\
\hline $\mathbf{T}_{5}$ & $\begin{array}{l}\text { Nirgudi } \\
(V . \text { negundo })\end{array}$ & 34.00 & 31.00 & 27.33 & $\begin{array}{c}62.39 \\
(38.60)\end{array}$ & $\begin{array}{c}65.55 \\
(40.95)\end{array}$ & $\begin{array}{c}69.62 \\
(44.12)\end{array}$ \\
\hline $\mathrm{T}_{6}$ & $\begin{array}{l}\text { Neem leaf extract } \\
\text { (A. indica) }\end{array}$ & 43.00 & 35.66 & 30.66 & $\begin{array}{c}52.22 \\
(31.47)\end{array}$ & $\begin{array}{c}60.37 \\
(37.13)\end{array}$ & $\begin{array}{c}65.92 \\
(41.24)\end{array}$ \\
\hline $\mathbf{T}_{7}$ & $\begin{array}{l}\text { Garlic } \\
\text { (A. sativum) }\end{array}$ & 19.66 & 14.33 & 10.33 & $\begin{array}{c}78.14 \\
(51.38)\end{array}$ & $\begin{array}{c}84.07 \\
(57.23)\end{array}$ & $\begin{array}{c}88.51 \\
(62.28)\end{array}$ \\
\hline $\mathbf{T}_{8}$ & $\begin{array}{l}\text { Turmeric } \\
(\text { C. longa })\end{array}$ & 61.33 & 58.33 & 56.00 & $\begin{array}{l}31.85 \\
(18.57)\end{array}$ & $\begin{array}{l}35.14 \\
(20.59)\end{array}$ & $\begin{array}{c}37.77 \\
(22.19)\end{array}$ \\
\hline $\mathrm{T}_{9}$ & $\begin{array}{l}\text { Adulsa } \\
\text { (A. vasica) }\end{array}$ & 82.00 & 78.66 & 75.33 & $\begin{array}{c}8.88 \\
(5.09)\end{array}$ & $\begin{array}{l}12.59 \\
(7.23)\end{array}$ & $\begin{array}{l}16.29 \\
(9.37)\end{array}$ \\
\hline $\mathrm{T}_{10}$ & $\begin{array}{l}\text { Shatawari } \\
\text { (A.racemosa) }\end{array}$ & 79.66 & 73.66 & 70.66 & $\begin{array}{l}11.48 \\
(6.59)\end{array}$ & $\begin{array}{c}18.14 \\
(10.45)\end{array}$ & $\begin{array}{c}21.48 \\
(12.40)\end{array}$ \\
\hline $\mathrm{T}_{11}$ & $\begin{array}{l}\text { Noni leaf extract } \\
\text { (M.citrifolia) }\end{array}$ & 82.00 & 76.33 & 71.00 & $\begin{array}{c}8.88 \\
(5.09)\end{array}$ & $\begin{array}{l}15.18 \\
(8.73)\end{array}$ & $\begin{array}{c}21.11 \\
(12.18)\end{array}$ \\
\hline $\mathbf{T}_{12}$ & Control (untreated) & 90.00 & 90.00 & 90.00 & $\begin{array}{l}00.00 \\
(0.00)\end{array}$ & $\begin{array}{l}00.00 \\
(0.00)\end{array}$ & $\begin{array}{l}00.00 \\
(0.00)\end{array}$ \\
\hline & $\mathbf{S E} \pm$ & 0.45 & 0.43 & 0.40 & 0.45 & 0.52 & 0.52 \\
\hline & $\mathrm{CD}(\mathrm{P}=0.05)$ & 1.31 & 1.26 & 1.27 & 1.32 & 1.52 & 1.53 \\
\hline
\end{tabular}

* Mean of three replications,

Col. $=$ Colony, Dia. $=$ Diameter, Conc.$=$ Concentration,

Av. =Average, Figures in parenthesis are arc sine transformed values. 
Table.2 Efficacy of organic amendments (pre sowing) against $M$. phaseolina, causing dry root rot in safflower cv. Bhima (pot culture)

\begin{tabular}{|c|c|c|c|c|c|c|c|c|}
\hline \multirow[t]{2}{*}{$\begin{array}{l}\text { Tr. } \\
\text { No. }\end{array}$} & \multirow[t]{2}{*}{ Treatments } & \multirow[t]{2}{*}{$\begin{array}{c}\text { Germination } \\
(\%)^{*}\end{array}$} & \multicolumn{2}{|c|}{$\begin{array}{c}\text { Rot/ mortality } \\
(\%) *\end{array}$} & \multirow{2}{*}{$\begin{array}{c}\text { Average } \\
\text { mortality } \\
(\%)\end{array}$} & \multicolumn{2}{|c|}{$\begin{array}{l}\text { Reduction over } \\
\text { control }(\%)^{*}\end{array}$} & \multirow{2}{*}{$\begin{array}{c}\text { Average } \\
\text { reduction } \\
(\%)\end{array}$} \\
\hline & & & PESR & PESM & & PESR & PESM & \\
\hline$T_{1}$ & Neem cake & $\begin{array}{c}85.00 \\
(67.21)\end{array}$ & $\begin{array}{l}15.00 \\
(22.79)\end{array}$ & $\begin{array}{l}22.66 \\
(28.43)\end{array}$ & $\begin{array}{c}18.33 \\
(25.72)\end{array}$ & $\begin{array}{c}78.57 \\
(62.42)\end{array}$ & $\begin{array}{c}70.03 \\
(56.51)\end{array}$ & $\begin{array}{c}74.30 \\
(59.59)\end{array}$ \\
\hline$\overline{T_{2}}$ & Castor cake & $\begin{array}{c}56.60 \\
(34.57)\end{array}$ & $\begin{array}{l}43.33 \\
(25.71)\end{array}$ & $\begin{array}{c}42.22 \\
(25.48)\end{array}$ & $\begin{array}{c}42.77 \\
(25.59)\end{array}$ & $\begin{array}{c}31.74 \\
(18.51)\end{array}$ & $\begin{array}{c}29.62 \\
(19.14)\end{array}$ & $\begin{array}{c}30.68 \\
(18.82)\end{array}$ \\
\hline $\mathbf{T}_{\mathbf{3}}$ & $\begin{array}{l}\text { Groundnut } \\
\text { cake }\end{array}$ & $\begin{array}{c}71.61 \\
(57.84)\end{array}$ & $\begin{array}{c}28.33 \\
(32.16)\end{array}$ & $\begin{array}{c}34.92 \\
(36.22)\end{array}$ & $\begin{array}{c}31.63 \\
(34.22)\end{array}$ & $\begin{array}{c}59.53 \\
(50.49)\end{array}$ & $\begin{array}{c}55.61 \\
(48.22)\end{array}$ & $\begin{array}{c}57.57 \\
(49.35)\end{array}$ \\
\hline $\mathbf{T}_{4}$ & Cotton cake & $\begin{array}{c}80.00 \\
(63.43)\end{array}$ & $\begin{array}{l}20.00 \\
(26.57)\end{array}$ & $\begin{array}{c}29.11 \\
(32.61)\end{array}$ & $\begin{array}{c}24.56 \\
(29.71)\end{array}$ & $\begin{array}{c}71.43 \\
(57.69)\end{array}$ & $\begin{array}{c}63.00 \\
(52.54)\end{array}$ & $\begin{array}{c}67.22 \\
(55.07)\end{array}$ \\
\hline $\mathbf{T}_{5}$ & $\begin{array}{l}\text { Sunflower } \\
\text { cake }\end{array}$ & $\begin{array}{c}65.00 \\
(53.73)\end{array}$ & $\begin{array}{c}35.00 \\
(36.27)\end{array}$ & $\begin{array}{c}38.46 \\
(38.43)\end{array}$ & $\begin{array}{c}36.73 \\
(37.30)\end{array}$ & $\begin{array}{c}50.00 \\
(45.00)\end{array}$ & $\begin{array}{c}51.11 \\
(45.64)\end{array}$ & $\begin{array}{c}50.56 \\
(45.32)\end{array}$ \\
\hline$T_{6}$ & $\begin{array}{l}\text { Fym / } \\
\text { Compost }\end{array}$ & $\begin{array}{c}41.67 \\
(40.20)\end{array}$ & $\begin{array}{c}58.33 \\
(49.80)\end{array}$ & $\begin{array}{c}60.19 \\
(58.88)\end{array}$ & $\begin{array}{c}59.26 \\
(50.54)\end{array}$ & $\begin{array}{l}16.67 \\
(24.10)\end{array}$ & $\begin{array}{c}23.51 \\
(29.00)\end{array}$ & $\begin{array}{c}20.09 \\
(26.63)\end{array}$ \\
\hline$\overline{T_{7}}$ & $\begin{array}{l}\text { Poultry } \\
\text { manure }\end{array}$ & $\begin{array}{c}58.33 \\
(49.80)\end{array}$ & $\begin{array}{c}41.67 \\
(40.20)\end{array}$ & $\begin{array}{c}45.71 \\
(42.54)\end{array}$ & $\begin{array}{c}43.69 \\
(41.37)\end{array}$ & $\begin{array}{c}40.47 \\
(39.51)\end{array}$ & $\begin{array}{c}41.90 \\
(40.34)\end{array}$ & $\begin{array}{c}41.19 \\
(39.95)\end{array}$ \\
\hline$\overline{T_{8}}$ & Vermicompost & $\begin{array}{c}55.00 \\
(47.87)\end{array}$ & $\begin{array}{c}45.00 \\
(42.13)\end{array}$ & $\begin{array}{c}48.48 \\
(44.13)\end{array}$ & $\begin{array}{c}46.47 \\
(43.13)\end{array}$ & $\begin{array}{c}35.71 \\
(42.13)\end{array}$ & $\begin{array}{c}38.38 \\
(38.28)\end{array}$ & $\begin{array}{c}37.04 \\
(40.42)\end{array}$ \\
\hline$T_{9}$ & $\begin{array}{l}\text { Control } \\
\text { (untreated) }\end{array}$ & $\begin{array}{c}30.00 \\
(21.53)\end{array}$ & $\begin{array}{c}70.00 \\
(56.79)\end{array}$ & $\begin{array}{c}78.68 \\
(62.50)\end{array}$ & $\begin{array}{c}74.34 \\
(59.60)\end{array}$ & $\begin{array}{c}00.00 \\
(00.00)\end{array}$ & $\begin{array}{c}00.00 \\
(00.00)\end{array}$ & $\begin{array}{c}00.00 \\
(00.00)\end{array}$ \\
\hline & S.E. \pm & 3.01 & 3.01 & 3.61 & 3.31 & 3.69 & 3.61 & 3.65 \\
\hline & C.D. $(P=0.05)$ & 8.78 & 8.78 & 10.53 & 9.62 & 10.77 & 10.53 & 10.65 \\
\hline
\end{tabular}

*-Mean of three replications, PESR- Pre emergence seed rot, PESM- Post emergence seedling mortality, Figures in parentheses are arc sine transformed values. 


\section{PLATE I}

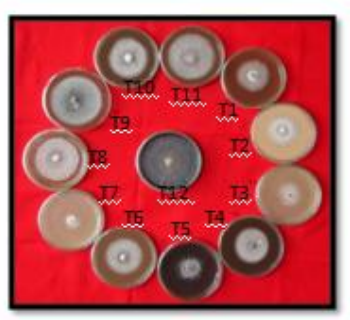

$10 \%$

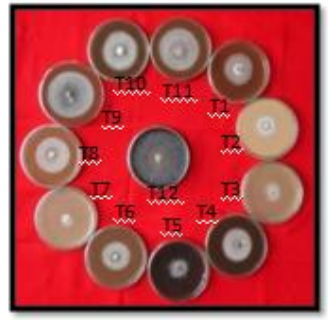

$15 \%$

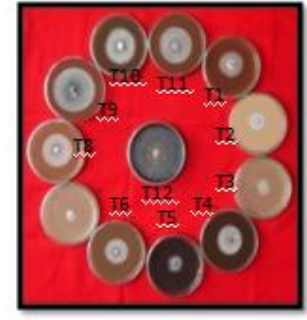

$20 \%$
$\mathrm{T}_{1}$ : Mehandi (L.innermis)

$\mathrm{T}_{2}$ : Ginger (Z. officünale)

$\mathrm{T}_{3}$ : Onion (A. cepa)

$\mathrm{T}_{4}:$ Tulsi $(O$. sanctum $)$

$T_{5}$ : Nirgudi $(V \cdot$ negundo)

$\mathrm{T}_{6}: \operatorname{Neem}($ A. indica $)$
$\mathrm{T}_{7}$ : Garlic (A. sativum)

$\mathrm{T}_{8}$ : Turmeric $($ C. longa $)$

$\mathrm{T}_{9}:$ Adulsa (A. vasica)

$\mathrm{T}_{10}$ : Shatavari (A. racemosa)

$T_{11}:$ Noni (M. citrofolia)

$\mathrm{T}_{12}$ : Control

In vitro efficacy of the botanicals at 10,15 and $20 \%$ conc against mycelial growth and inhibition of $\boldsymbol{M}$. phaseolina.

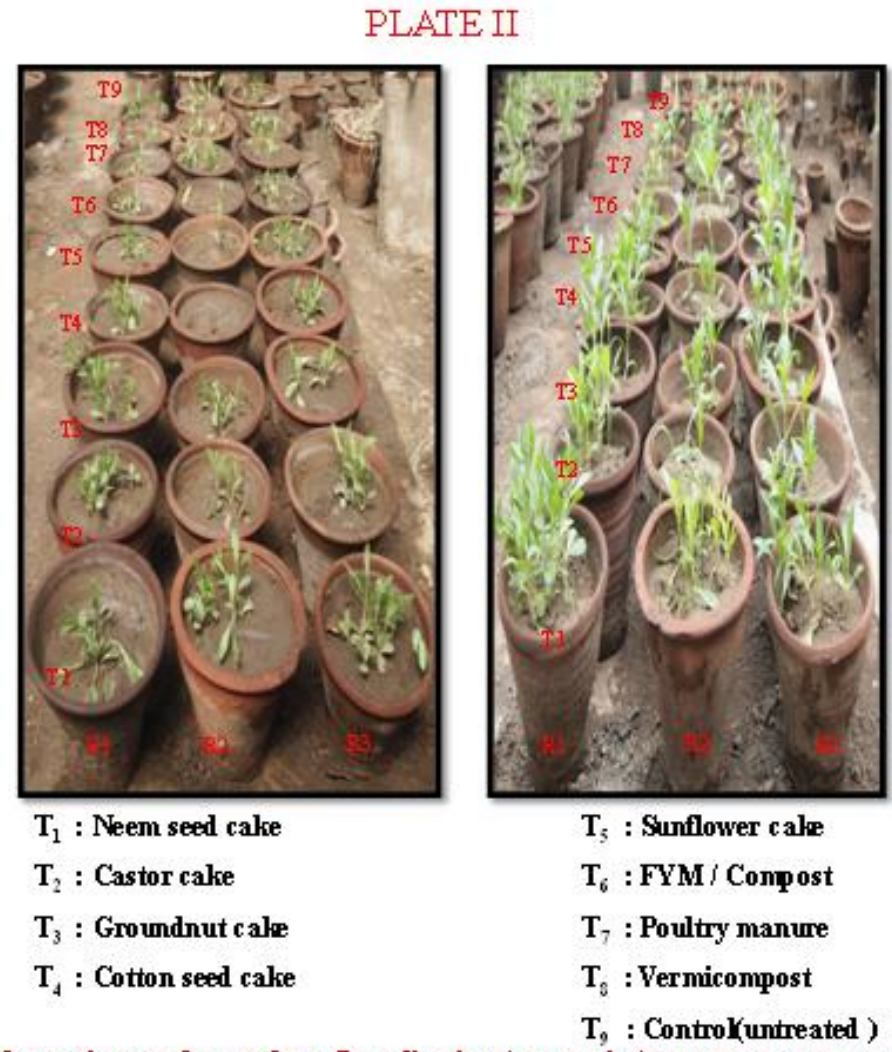

Effect of organic amendments by soil application (pre-sowing) on pre- emergence seed rot (A) and post-emergence seedling mortaliny (B) caused by $M$. phase olina in safflower cv. Bhima 
Fig.1 In vitro efficacy of botanicals at $10 \%$ against mycelial growth and inhibition of $M$. phaseolina

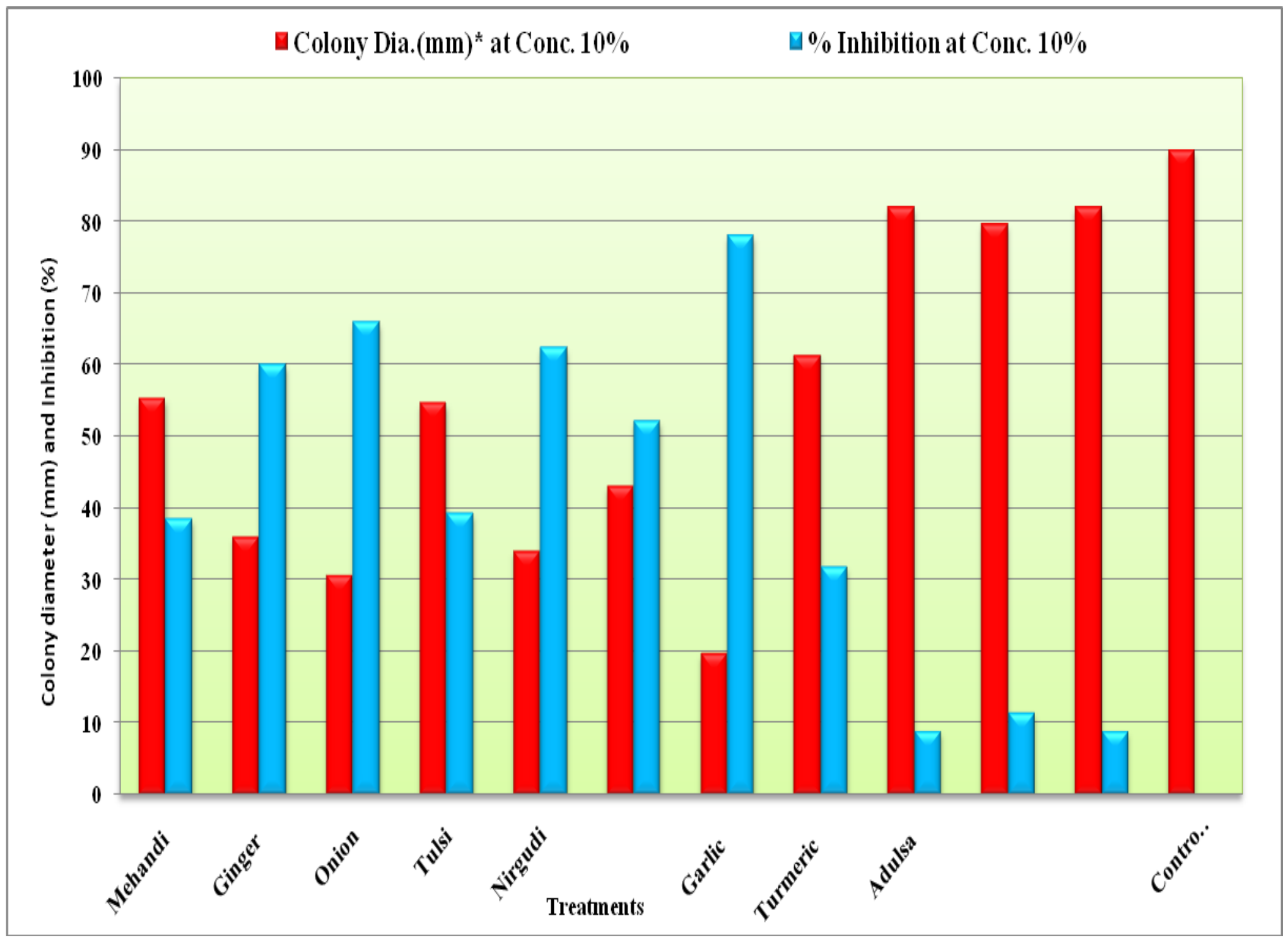


Fig.2 In vitro efficacy of botanicals at $15 \%$ against mycelial growth and inhibition of $M$. phaseolina

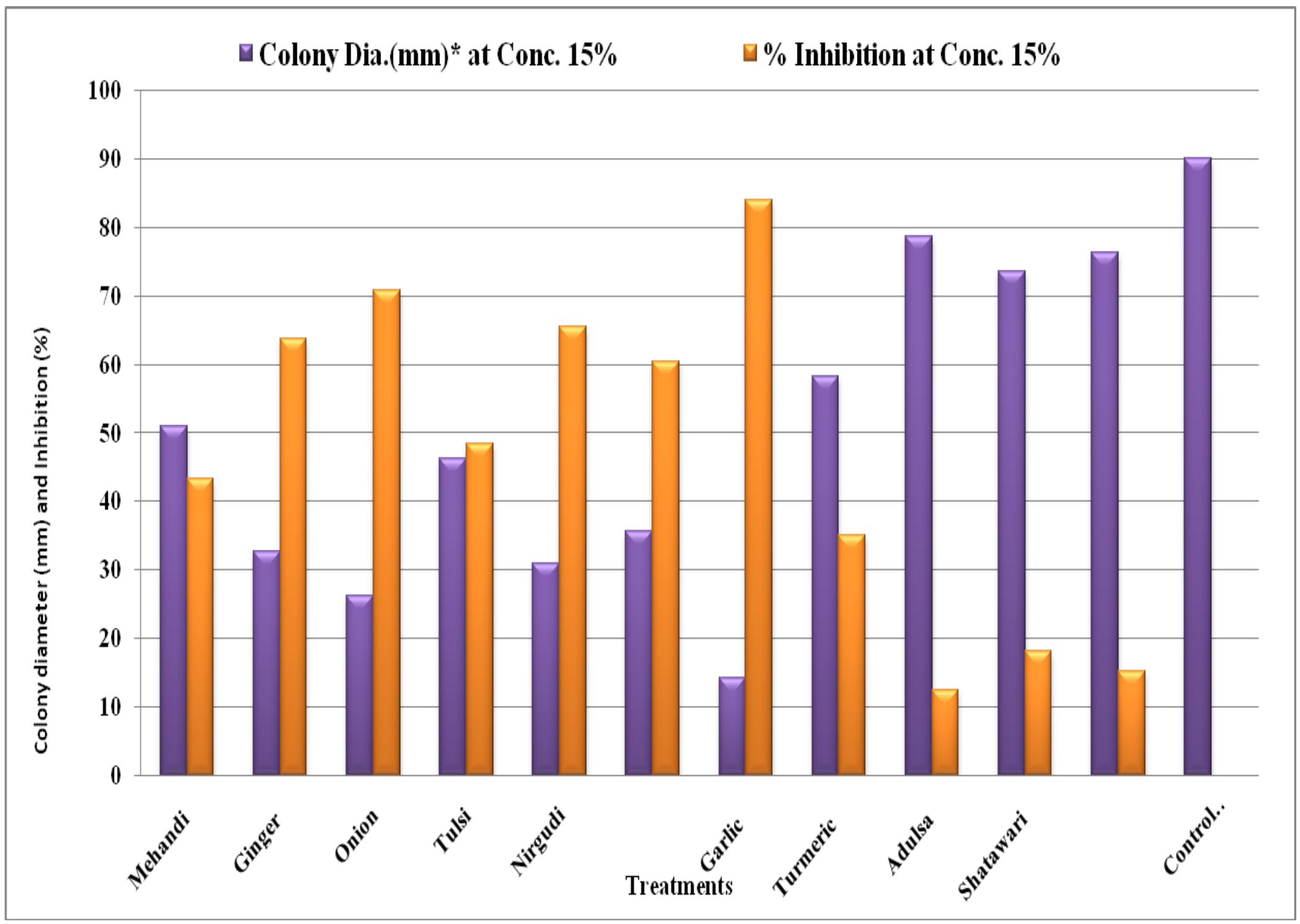


Fig.3 In vitro efficacy of botanicals at $20 \%$ against mycelial growth and inhibition of $M$. phaseolina

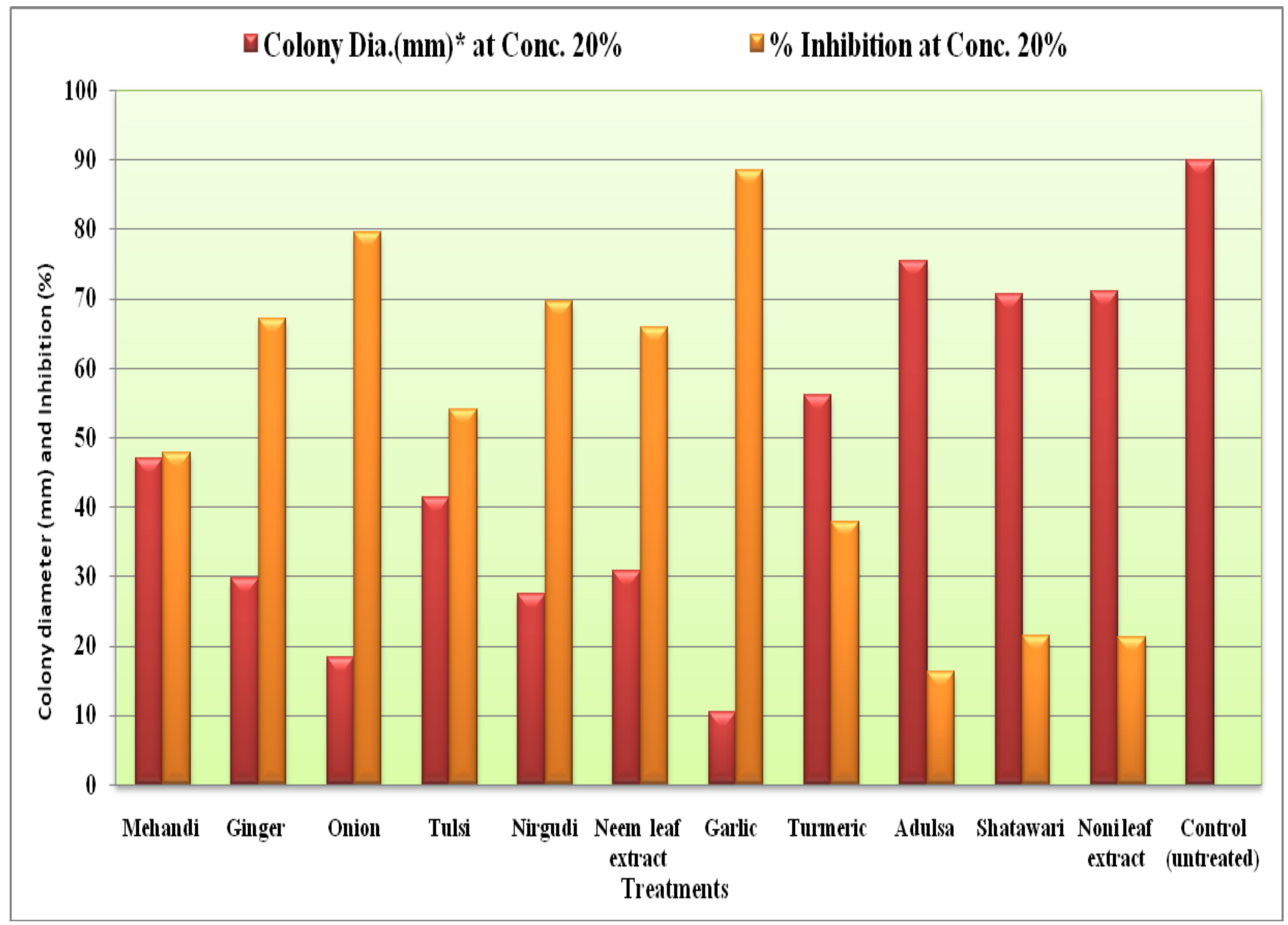


Fig.4 Efficacy of organic amendments soil application (pre sowing) against M. phaseolina, causing dry root rot in safflower cv. Bhima (pot culture)

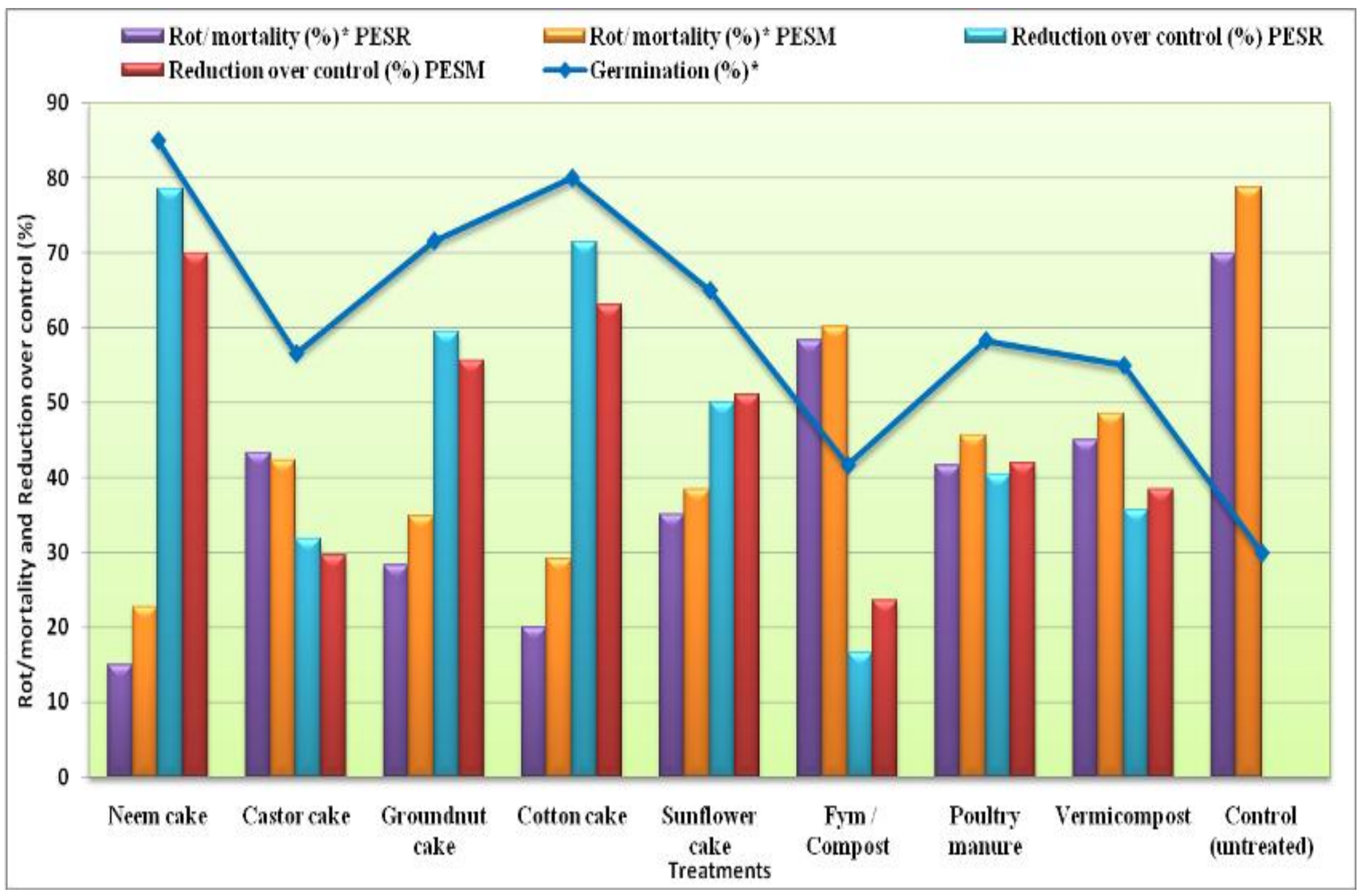


Results of the present study on antifungal activity of the botanicals are in conformity with those reported earlier by several workers. Botanicals/plant extracts viz., A. sativum, A. cepa, V. negundo, Z. officinale, A. indica, $O$. sanctum, L. innermis, $C$. longa, and $A$. racemosa. were reported antifungal/fungistatic against $M$. phaseolina, earlier by several workers (Sundarraj et al., 1996; Datar, 1999; Jha et al., 2000; Sharma et al., 2003; Tandel et al., 2010; Magar et al., 2011; Javid and Saddique, 2012; Lakpale, 2012; Amreen and Sanathkumar, 2013 and Dhingani et al., 2013).

\section{In vitro evaluation of organic amendments}

A total of 08 amendments were evaluated as pre-sowing application against $M$. phaseolina (sick soil method), sowing susceptible safflower cv. Bhima in pot culture under screen house conditions. The results obtained on percentage seed germination, preemergence seed rot (PESR) and postemergence seedling mortality (PESM) are presented in the Table 2 and depicted in Figure 4 and PLATE-II.

\section{Effect on seed germination}

Result (Table 2, Fig. 4 and PLATE-II) revealed that all the test amendments significantly improved the percentage seed germination over untreated control (sick soil) and it was ranged from 41.67 to 85.00 per cent, as against 30.00 per cent in untreated control (sick soil).

However, Neem seed cake was found most effective with significantly highest seed germination $(85.00 \%)$. This was followed by the amendments viz., Cotton seed cake (80.00\%), Groundnut cake (71.61\%), Sunflower cake $(65.00 \%)$, Poultry manure $(58.33 \%)$ and Castor cake $(56.60 \%)$. However, Vermicompost and FYM were found least effective with comparatively minimum seed germination of 55.00 and 41.67 per cent, respectively.

\section{Effect on pre- and post-emergence} mortality

Results (Table 2, Fig. 4 and PLATE-II) revealed that all the test amendments significantly influenced both pre-emergence seed rot (PESR) and post emergence seedling mortality (PESM), caused by $M$. phaseolina in safflower cv. Bhima. The pre-emergence seed rot (PESR) recorded with all the test amendment was ranged from 15.00 to 58.33 per cent, as against 70.00 per cent in untreated control (sick soil). However, significantly least pre-emergence seed rot was recorded with Neem seed cake (15.00\%). This was followed by the amendments viz., Cotton seed cake $(20.00 \%)$, Groundnut cake (28.33\%), Sunflower cake $(35.00 \%)$, Poultry manure (41.67\%), and Castor cake (43.33\%). However, Vermicompost and FYM were found least effective with comparatively maximum PESR of 45.00 and 58.33 per cent, respectively.

Similar trend in respect of the postemergence seedling mortality (PESM) was also observed and it was ranged from 22.66 to 60.19 per cent, as against 78.68 per cent in untreated control (sick soil). Of the amendments tested, Neem seed cake found most effective with significantly least postemergence seedling mortality 22.66 per cent.

This was followed by the amendments viz., Neem seed cake $(22.66 \%)$, Cotton seed cake (29.11\%), Groundnut cake (34.92\%), Sunflower cake $(38.46 \%)$, Poultry manure $(45.71 \%)$, and Castor cake $(42.22 \%)$. However, Vermicompost and FYM were found least effective with comparatively maximum PESM of 48.48 and 60.19 per cent, respectively. 
The average mortality recorded with all the test amendments was ranged from 18.33 to 59.26 per cent, as against 74.34 per cent in untreated control (sick soil). However, significantly least average mortality was recorded with Neem seed cake (18.33\%), followed by the amendments viz., Cotton seed cake (24.34\%), Groundnut cake (31.63\%), Sunflower cake $(36.73 \%)$, Poultry manure $(43.69 \%)$, Castor cake $(42.77 \%)$. Whereas, Vermicompost and FYM were found least effective with comparatively maximum average mortality of 46.47 and 57.50 per cent, respectively.

\section{Reduction in mortality}

All the test amendments were found to reduce/ control both the mortalities (pre and post), over untreated control (Table 2). The percentage reduction of the pre- and postemergence mortalities were ranged from 16.67 (FYM) to 78.57 (Neem seed cake) per cent and 23.51 (FYM) to 70.03 (Neem seed cake) per cent, respectively. All the amendment tested significantly highest reduction in preemergence seed rot $(78.57 \%)$ and postemergence seedling mortality $(70.03 \%)$ were recorded with Neem seed cake. These were followed by the amendments viz., Cotton seed cake (71.43 and 63.00\%), Groundnut cake (59.53 and 55.61\%), Sunflower cake (50.00 and $51.11 \%$ ) Poultry manure (40.47 and $41.90 \%)$, Castor cake (31.74 and 29.62\%). Vermicompost (35.71 and 38.38\%). The soil amendment with FYM was found least effective with significantly least reduction in PESR (16.67\%) and PESM (23.51\%).

The average mortality reduction (PESR and PESM) recorded with all the test amendments was ranged from 20.09 (FYM) to 74.30 (Neem seed cake) per cent over untreated control. However, significantly highest average mortality reduction $(74.30 \%)$ was recorded with Neem seed cake. This was followed by the amendments viz., Cotton seed cake $(67.22 \%)$ Groundnut cake (57.57\%), Sunflower cake (50.56) Less than 45.00 per cent average mortality reduction was recorded with Poultry manure (41.19\%), Vermicompost (37.04\%) Castor cake (30.68); and whereas, FYM was found least effective with significantly least $(20.09 \%)$ reduction in average mortality.

Results of the present study obtained on the efficacy of the soil amendments against $M$. phaseolina are in conformity with those reported earlier by several workers. Organic amendments viz., Neem seed cake, Cotton seed cake, Groundnut cake, Sunflower cake, Poultry manure, Vermicompost and FYM were reported antifungal/fungistatic against M. phaseolina, earlier by several workers (Muthuswami and Mirippan, 1991; Hundekar et al., 1998; Sudha and Prabhu, 2008; Jaiman et al., 2009; Mehta et al., 2010).

\section{References}

Amar singh and T.P. Bhowmik (1979). Occurance of charcoal rot of safflower in India. Indian Phytopath, 32:626-627.

Amreen, T. and Sanathkumar, V. B. (2013). Sensitivity of Macrophomina phaseolina (Tassi) Goid, causing collar rot of maize against antagonists, plant extracts and animal products. Res. J. Agril. Sci. 4(3): 372-374.

Das, I. K., Indira, S., Annapurna, A., Prabhakar, B. and Seetharama, N. (2008). Biocontrol of charcoal rot in sorghum by Pseudomonas fluorescens associated with the rhizosphere. Crop Prot. 27:1407-1414.

Datar, V.V. (1999). Efficacy of plant extracts against $M$. phaseolina (Tassi) Goid, the incitant of charcoal rot of sorghum. J. Mycol. Pl. Pathol. 29 (2): 261-253.

Dhingani, J. C., Solanky, K. U. and Kansara, S. S. (2013). Management of root rot 
disease [M. phaseolina (Tassi) Goid.] of chickpea through botanicals and oil cakes. An. Intern. Quar. J. Life sci. 8(3): 739-742.

Hazarika, D. K. and Das, K. K. (1998). Biological management of root rot of French bean (Phaseolus vulgaris L.), caused by Rhizoctonia solani. Pl. Dis. Res. 13 (2): 101-105.

Hundekar, A.R., Anahosur, K.H., Patil, M.S., Kalappanavar, I.K. and Chattannavar, S.N. (1998). In vitro evaluation of organic amendments against stalk rot of sorghum. J. Mycol. Pl. Pathol. 28(1): 26-30.

Jahagirdar, S., Patil, M. S. and Indra, S. (2001). Biological control of charcoal rot of sorghum caused by $M$. phaseoina (Tassi) Goid. Agril. Sci. Digest. 21 (3): 153-156.

Jaiman, R.K.; Jain S. C. and Pankaj Sharma (2009). Field evalution of fungicides, bioagents and soil amendments against root rot caused by Macrophomina phaseolina in Cluster bean.J. Mycol. PL. Paythol. 39(1); 74-76.

Javaid, A. and Saddique, A. (2012). Control of charcoal rot fungus Macrophomina phaseolina by extracts of Daturametel. Natural Product Research. 26 (18): 1715-1720.

Jha, A. K., Dubey, S. C. and Jha, D. K. (2000). Evaluation of different leaf extracts and oil cakes against Macrophomina phaseolina, causing collar rot of okra. J. Res. BirsaAgril. Univ. 12 (2): 225-228.

Kaur, S., Singh, N. and Sandhu, P. S. (2008). In vitro evaluation of Trichoderma viride and $T$. harzianum against $M$. phaseolina, causing charcoal root rot of sunflower. Pl. Dis. Res. 25 (1): 79.

Kore, S. S. and R. W. Deshmukh (1982). Charcoal rrot rot of safflower caused by Macrophomina phaseolina (Tassi) Goid
Res. Bull. Marathwada Agril. Univerc., 6:43-45.

Kumari, R., Shekhawat, K. S., Gupta, R. and Khokhar, M. K. (2012). Integrated management against root-rot of Mungbean [Vigna radiata (L.) Wilczek], incited by Macrophomina phaseolina. J. Pl. Pathol. Microbiol.3:136.

Lakpale, N. (2012). Efficacy of inhibitors against Macrophomina phaseolina, inciting stolon rot of mint. Ann. Pl. Prot. Sci. 20 (1): 242-243.

Lambhate, S. S., Chaudhari, G. K., Mehetre, S. S. and Zanjare, S R. (2002). In vitro evaluation of chemicals against root rot of cotton caused by Macrophomina phaseolina. J. Mah. Agric. Univ. 27 (1): 99-100.

Lokesha, N. M. and Benagi, V. I. (2007). Biological management of pigeonpea Dry root rot caused by Macrophomina phaseolina. Karnataka J. Agric. Sci.20 (1): $54-56$.

Lukade, G. M. (1992). Effect of organic soil amendments on root rot incidence of safflower. Madras Agric. J., 79 (3): 179181.

Magar, S. V., Kadam, J. J., Rite, S. C., Thaware, D. S. and Potphode, P. D. (2011). Exploration of plant extracts and fungal antagonists against Macrophomina phaseolina (Tassi.) Goid., causing leaf spot in greengram. Int. J. Pl. Prot. 4 (1): 30-33.

Malathi S. and S. Doraisamy (2003). Compatibility of $T$. harzianum with fungicides against $M$. phaseolina $\mathrm{pl}$. Dis. Res. 18 (2): 139-143.

Meena, S. and Sangit, K. (2010). Potantial biocontrol agents for the management of $M$. Phaseolina incident of charcoal rot in maize. Arch. Phytopathol. Pl.Prot. 43 (4): 379-383.

Meena, S., Shrama, R. C., Lokendra, S. and Ram, D. (2006).Morphological and 
pathogenic variablity of Macrophomina phaseolina (Tassi) Goid. incident of chorcol rot of maize in India. Indian Phytopath. 59 (3): 294-298.

Mehta, N., Hiea, N. T. and Sangwan, M. S. (2010). Management of white stem rot (Sclerotiniascle rotiorum) of mustard with organic soil amendments. J. Mycol. Pl. Pathol. 40 (2): 238-243.

Moradia, A. M. and Khandar, R. R. (2011). Losses of yield of groundnut due to dry root rot (M. phaseolina) and their management under in vivo conditions. Int. J. Agril. Sci. 7(2): 282-285.

Muthuswmi, S. and Mariappan, V. (1991). Disintegration of sclerotia of M.phaseolina (Soybean isolate) by oil cake extracts. Indian Phytopath. 26 (12) 271-273.

Nene, Y. L. and P. N. Thapliyal (1993). Fungicides in Plant Disease Control. $3^{\text {rd }}$ Ed. Oxford IBH Publ. New Delhi. pp: 507.

Pan, S. and Bhagat, S. (2007). Antagonistic potential of Trichoderma and Gliocladium spp. from West Bengal. J. Mycol. Pl. Pathol. 31 (2): 235-239.

Shambharkar, D. A. and D. V. Indi (1987). Causal organism of safflower diseases and their importance. AICRP on Oilseeds (Safflower), Solapur, Maharashtra, India.pp: 181.

Shrishail, M., Vastrad, B.D., Biradar, S.S., Karbhantnal S.Y., Wali and Arun, S. (2012). In vitro evaluation of bioagents for the management of charcoal rot of sorghum. In: Souvenior of $3^{\text {rd }}$ Global Conference (ISMPP) on "Plant
Pathology for Food Security", held at MPUAT, Udaipur, January 10-13, pp.140.

Shrivastava, S. N. and Tripathi, R. C. (1998). Management of sugarbeet seedling disease complex by combination of fungicides. Indian Phytopath. 51(1): 7577.

Singh, S. N.; Shrivastav, S. K. and M. N. Khare (1987). Effect of varying nitrogen doses and dates of sowing on the incidence of root rot (Rhizoctonia bataticola) and leaf spot (Alternaria carthami) diseases of safflower. J. Mycol. Pl. Pathol., 17(3): 351-352.

Sudha A. and Prabhu S. (2008). Evaluation of organic soil amendments against $M$. phaseolina biopesticides, 1 (2): 143145.

Sundarraj, T. V., Kurucheve J. and Jayaraj (1996). Screening of higher plants and animal faeces for the fungitoxicity against Rhizoctonia solani. Indian Phytopath. 49 (4): 398-403.

Suryawanshi, V. P. Hajare, S. T., Karnewar, S. D. and Kamble, N. S. (2008). In vitro chemical and biological control of Macrophomina phaseolina on black gram. J. Soils Crops. 18 (2): 375-378.

Tandel, D. B., Sabalpara, A.N. and Pandya, J.R. (2010). Efficacy of fungicides and phytoextrats against green gram leaf blight caused by Macrophomina phaseolina. J. Pl. Dis. Sci. 5(1):48-50.

Vincent, J.M. (1927). Distortion of fungal hyphae in the presence of certain inhibitors, Nature: 159-180.

\section{How to cite this article:}

Gawande, P.N., V.M. Gholve and Ghuge, S.B. 2018. In vitro Bio-Efficacy of Botanicals and Organic Amendments against Dry Root Rot of Safflower. Int.J.Curr.Microbiol.App.Sci. 7(11): 2048-2062. doi: https://doi.org/10.20546/ijcmas.2018.711.233 